\title{
Modelado dinámico del manipulador serial Mitsubishi Movemaster RV-M1 usando SolidWorks
}

\section{Dynamic modelling of the Mitsubishi Movemaster RV-M1 serial manipulator using SolidWorks}

\author{
A. Barraza ${ }^{1}$, J.C. Rúa ${ }^{2}$, J.L. Sosa ${ }^{3}$, J. Díaz ${ }^{4}$, E. Yime ${ }^{5}$, J. Roldán ${ }^{6}$ \\ ${ }^{1}$ Ing. Mec., Uniatlántico, Colombia, aljobaca@gmail.com; ${ }^{2}$ Ing. Mec., Uniatlántico, Colombia, juansel17@ hotmail.com; \\ ${ }^{3}$ Ing. Mec., Uniatlántico, Colombia, jlsosarodriguez@ hotmail.com; ${ }^{4}$ Dpto. Ing. Mec., Univ. George Mason, EEUU, \\ jdiazgon@masonlive.gmu.edu; ${ }^{5}$ Ing. Mecatr., UTB, GIMAT, Colombia, eyime@ unitecnologica.edu.co; ${ }^{6}$ Ing. Mec., Uniatlántico, \\ DIMER, Colombia, javierroldan@mail.uniatlantico.edu.co
}

Recibido: dic 5, 2015. Aceptado: jun 15, 2016. Versión final: jun 15, 2016

\begin{abstract}
RESUMEN
Se presenta la aplicación del principio energético Lagrange-Euler para obtener el modelo matemático de la dinámica del robot Mitsubishi Movemaster RV-M1. La determinación de la ubicación de los centros de masa de cada eslabón y las matrices de inercia se realiza a partir de un modelo CAD generado con el software SolidWorks, en el cual se tuvo en cuenta los elementos internos de cada eslabón con su material y ubicación. El modelo matemático se usa para calcular los torques requeridos en una estrategia de control de compensación por gravedad, y se compara el efecto de las propiedades obtenidas con SolidWorks en la respuesta del robot con aquella obtenida utilizando parámetros reportados en literatura existente. Se lograron resultados satisfactorios en los errores del espacio de la tarea mediante este enfoque, no experimental pero detallado, para determinar las propiedades dinámicas de un robot usando un software muy común en los programas de Ingeniería Mecánica de las universidades colombianas.
\end{abstract}

Palabras clave: dinámica, Movemaster RV-M1, robótica, SolidWorks.

\begin{abstract}
This paper presents how the mathematical model of the Mitsubishi RV-M1 robot dynamics is obtained from the application of the Lagrange-Euler energy principle. A SolidWorks CAD model of the robot was created, where the internal elements material and location were considered for each link. This detailed CAD model was used in the attainment of the centers of mass and the inertia matrixes of each link. The gravity compensation control law was simulated for the robot, and the mathematical dynamic model was used to calculate the control torques. The results obtained for the SolidWorks generated values were compared against those using dynamic properties in available literature. Satisfactory results were obtained in the workspace error by using an approach that is very detailed although non-experimental, supported with a software that is widely used in the Mechanical Engineering Programas of the Colombian universities.
\end{abstract}

Keywords: dynamics, Movemaster RV-M1, robotics, SolidWorks.

\section{INTRODUCCIÓN}

Debido a las exigencias de calidad, seguridad y eficiencia que se han gestado en los sistemas de producción actuales, las industrias han optado por implementar tecnología para mejorar sus procesos. Uno de los avances tecnológicos ha sido la incursión de manipuladores robóticos en tareas repetitivas de precisión y gran riesgo para el ser humano [1], por lo que la Academia se ha apoyado en modelos que permitan la aplicación de todos los conceptos que encierra la robótica. Para tal fin, el manipulador Mitsubi shi Movemaster RV-M1 ha sido ampliamente usado como modelo para desarrollos teórico-prácticos, como se observa en [2;3]. Dentro de estos conceptos fundamentales, un modelado dinámico detallado permite una mejor estimación de las ganancias de las estrategias de control, y consecuentemente una implementación más sencilla [4].

Son pocas las investigaciones documentadas acerca del modelado dinámico explícito del manipulador RVM1. En el año 2010, en [5], se presentó la simulación del trabajo cooperativo de dos manipuladores RV-M1. En [5], un modelo CAD fue generado en SolidWorks y

Este artículo puede compartirse bajo la licencia CC BY-ND 4.0 (https://creativecommons.org/licenses/by-nd/4.0/) y debe referenciarse usando el siguiente formato: A. Barraza et al, "Modelado dinámico del manipulador serial Mitsubishi Movemaster RV-M1 usando SolidWorks," UIS Ingenierias, vol. 15, no. 2, pp. 49-62, jul-dic 2016, doi:10.18273/revuin.v15n2-2016004. 
exportado al software especializado [6] para crear un bloque integrado a Simulink de Matlab. En el trabajo [5], se consideró cada elemento del robot como un sólido, sin tener en cuenta la presencia de componentes internos como servomotores, cables y bandas, entre otros, los cuales afectan la ubicación de los centros de masa de los elementos, tal como se ilustra en la figura 1.

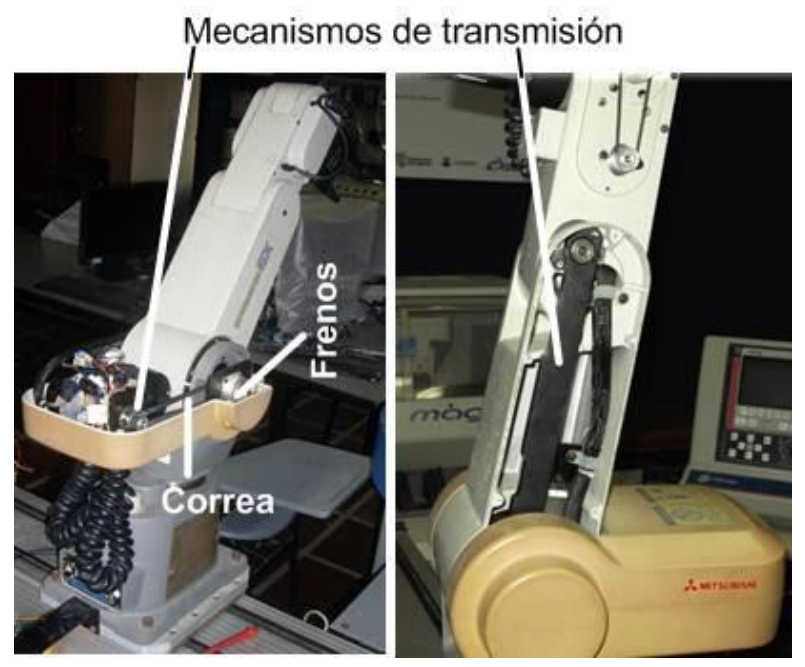

Figura 1. Componentes internos del robot RV-M1. Fuente. Elaboración propia.

Este artículo inicia con la descripción de la cinemática del manipulador RV-M1, para luego presentar su modelado dinámico. Se presenta el desarrollo de un modelo dinámico en el cual las propiedades de inercia de los elementos se hallaron mediante un modelo CAD hecho en SolidWorks, que considera la ubicación exacta, geometría y material de cada elemento interno de sus eslabones. Esos valores fueron utilizados para alimentar los parámetros de una planta en Simulink de Matlab, de manera que se pudiese simular una estrategia de control. Se compara el desempeño de un único controlador para dos modelos dinámicos: uno alimentado con los valores hallados en este trabajo y, el otro, con las propiedades utilizadas en [5]. Las simulaciones presentadas permiten la confrontación teórica de los resultados.

La relevancia de este trabajo se fundamenta en que los software especializados, como [6], en casos como las simulaciones de robots, no están comúnmente disponibles como recurso de base para los programas de Ingeniería Mecánica. Mientras que un software como SolidWorks es de amplio uso en tales programas para el desarrollo de diseño de maquinaria. Al utilizar este software para el modelado de robots, se está ampliando el campo de aplicación de esta herramienta computacional sin aumentar el costo de los proyectos relacionados. Como observan [7; 8; 9], un enfoque experimental para la identificación de parámetros requeriría de hardware especializado para la identificación de sistemas que permitan, por ejemplo, estimar parámetros adicionales como la fricción en juntas o envejecimiento del equipo.

\section{METODOLOGÍA}

\subsection{Cinemática de posición del manipulador Movemaster RV-M1}

Para que un manipulador realice una tarea específica, se debe establecer la posición y orientación de la herramienta y el posicionamiento relativo de cada eslabón. La metodología a utilizar para establecer los parámetros de referencia entre elementos del robot, es la teoría unificada de [10], la cual ha sido implementada con éxito en la cinemática de manipuladores seriales complejos, como el robot de seis eslabones [11], e inclusive en aplicaciones espaciales $[12 ; 13]$. De acuerdo con [14], los elementos tridimensionales se definen usando seis parámetros cinemáticos, que se muestran en la figura 2 y que son explicados a continuación.

- Vector de articulación $\mathbf{S}_{\mathrm{i}}$ : para juntas de rotación es un vector unitario que define la rotación, siguiendo la regla de la mano derecha.

- Vector del elemento $\mathbf{a}_{\mathrm{ij}}$ : vector unitario perpendicular a ambos vectores $\mathbf{S}_{\mathrm{i}}$ y $\mathbf{S}_{\mathrm{j}}$, sentido i-j.

- Distancia de articulación $\mathrm{S}_{\mathrm{i}}$ : distancia entre los vectores $\mathbf{a}_{\mathrm{hi}} \mathrm{y} \mathbf{a}_{\mathrm{ij}}$, medida a lo largo de $\mathbf{S}_{\mathrm{i}}$.

- Distancia del elemento $\mathrm{a}_{\mathrm{ij}}$ : distancia entre $\mathbf{S}_{\mathrm{i}}$ y $\mathbf{S}_{\mathrm{j}}$, medida a lo largo de $\mathbf{a}_{\mathrm{ij}}$.

- Ángulo de articulación $\theta_{\mathrm{i}}$ : ángulo medido con la regla de la mano derecha utilizando como base el vector de articulación $\mathbf{S}_{\mathrm{i}}$, y barrido de $\mathbf{a}_{\mathrm{hi}}$ a $\mathbf{a}_{\mathrm{ij}}$.

- Ángulo del elemento $\alpha_{\mathrm{ij}}$ : ángulo medido mediante la regla de la mano derecha utilizando como base en vector del elemento $\mathbf{a}_{\mathrm{ij}}$, y barrido de $\mathbf{S}_{\mathrm{i}}$ a $\mathbf{S}_{\mathrm{j}}$.

Los vectores de articulación y del elemento, que se muestran en la figura 2, establecen los sistemas coordenados asociados a cada elemento. El vector del elemento $\mathbf{S}_{\mathrm{i}}$ define la dirección del eje $\mathrm{z}$ del sistema coordenado $i, \mathrm{z}_{\mathrm{i}}$, asociado al elemento $i$ del manipulador. La dirección del eje $\mathrm{x}_{\mathrm{i}}$ es definida por el vector $\mathbf{a}_{\mathrm{ij}}$, y para definir un sistema coordenado cartesiano derecho, la dirección de eje yi se encuentra por medio del producto $\left(\mathbf{S}_{\mathrm{i}} \times \mathbf{a}_{\mathrm{ij}}\right) /\left|\mathbf{S}_{\mathrm{i}} \times \mathbf{a}_{\mathrm{ij}}\right|$. El subíndice $F$ en el sistema coordenado fijo $\left\{\mathrm{X}_{\mathrm{F}}-\mathrm{y}_{\mathrm{F}}-\mathrm{Z}_{\mathrm{F}}\right\}$ que aparece en la figura 2(d) denota el elemento fijo a tierra o marco inercial.

En la tabla I se muestran los valores de los parámetros cinemáticos tomados de [15] e ilustrados en la figura 3. La posición y la orientación relativas entre dos elementos se expresan mediante una matriz de transformación, de acuerdo con $[14 ; 16]$. Una matriz de transformación, ${ }_{j}^{\mathrm{i}} \mathrm{T}$, se crea con la composion de la matriz de rotación entre dos elementos consecutivos i e j, ${ }_{j}^{i} \mathrm{R}, \mathrm{y}$ del vector de posición del origen del marco de referencia del cuerpo $\mathrm{j}$ : 
$\left\{\mathrm{x}_{\mathrm{j}}-\mathrm{y}_{\mathrm{j}}-\mathrm{Z}_{\mathrm{j}}\right\}$, visto en el marco de referencia i: $\left\{\mathrm{x}_{\mathrm{i}}-\mathrm{y}_{\mathrm{i}}-\mathrm{Z}_{\mathrm{i}}\right\},{ }^{\mathrm{i}} \mathbf{P}_{0 \mathrm{j}}$, dispuestos como se presenta en la ecuación 1.

$$
{ }_{\mathrm{j}}^{\mathrm{i}} \mathrm{T}=\left[\begin{array}{cccc} 
& { }_{\mathrm{j}}^{\mathrm{i}} \mathrm{R} & { }^{\mathrm{i}} \mathbf{P}_{0 \mathrm{j}} \\
0 & 0 & 0 & 1
\end{array}\right]
$$

La nomenclatura empleada genera una matriz de transformación recurrente que relaciona dos elementos consecutivos del robot i e j, que está dada por la ecuación 2 , tomada de [14], donde $\mathrm{ci}=\cos (\theta \mathrm{i}), \mathrm{si}=\sin (\theta \mathrm{i}), \mathrm{cij}=$ $\cos (\alpha \mathrm{ij}), \operatorname{sij}=\sin (\alpha \mathrm{ij})$.

$$
{ }_{\mathrm{j}} \mathrm{T}=\left[\begin{array}{cccc}
c_{\mathrm{j}} & -\mathrm{s}_{\mathrm{j}} & 0 & \mathrm{a}_{\mathrm{ij}} \\
\mathrm{s}_{\mathrm{j}} \mathrm{c}_{\mathrm{ij}} & \mathrm{c}_{\mathrm{j}} \mathrm{c}_{\mathrm{ij}} & -\mathrm{s}_{\mathrm{ij}} & -\mathrm{s}_{\mathrm{ij}} \mathrm{S}_{\mathrm{j}} \\
\mathrm{s}_{\mathrm{j}} \mathrm{s}_{\mathrm{ij}} & \mathrm{c}_{\mathrm{j}} \mathrm{s}_{\mathrm{ij}} & \mathrm{c}_{\mathrm{ij}} & \mathrm{c}_{\mathrm{ij}} \mathrm{S}_{\mathrm{j}} \\
0 & 0 & 0 & 1
\end{array}\right]
$$

La matriz presentada en la ecuación 2 permite plantear matricialmente la cinemática del manipulador para relacionar la posición ${ }^{\mathrm{F}} \mathbf{P}_{\mathrm{H}}$, y orientación de la herramienta respecto al marco de referencia fijo $\left\{\mathrm{x}_{\mathrm{F}}-\mathrm{y}_{\mathrm{F}}-\mathrm{Z}_{\mathrm{F}}\right\}$, que se observa en la figura 4 , por medio del producto matricial de la ecuación 3, donde ${ }_{1}^{\mathrm{F}} \mathrm{T}$ es la matriz de transformación del primer elemento en el marco de referencia, ${ }_{j}^{\mathrm{i}} \mathrm{T}$ es la matriz de transformación del elemento j en el elemento $i$, y ${ }_{\mathrm{H}}^{5} \mathrm{~T}$ es la matriz de transformación de la herramienta ubicada en el último elemento del robot.

$$
{ }_{\mathrm{H}}^{\mathrm{F}} \mathrm{T}={ }_{1}^{\mathrm{F}} \mathrm{T}_{2}^{1} \mathrm{~T}_{3}^{2} \mathrm{~T}_{4}^{3} \mathrm{~T}_{5}^{4} \mathrm{~T}_{\mathrm{H}}^{5} \mathrm{~T}
$$

La aplicación recurrente de la ecuación 2 para hallar las matrices ${ }_{1}^{\mathrm{F}} \mathrm{T},{ }_{2}^{1} \mathrm{~T},{ }_{3}^{2} \mathrm{~T},{ }_{4}^{3} \mathrm{~T},{ }_{5}^{4} \mathrm{~T}$ y ${ }_{\mathrm{H}}^{5} \mathrm{~T}$, al ser reemplazadas en la Ecuación 3, generan la matriz de transformación del robot Mitsubishi RV-M1 en el marco fijo, dada en la ecuación 4.

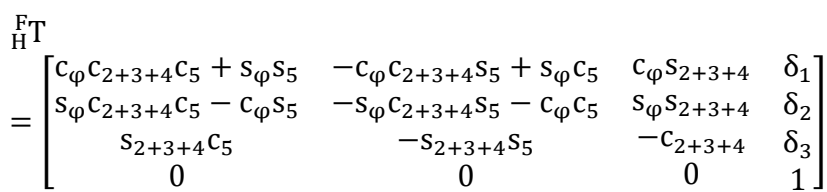

donde $\quad c_{\varphi}=\cos \left(\varphi_{1}\right), \quad s_{\varphi}=\sin \left(\varphi_{1}\right), \quad c_{2+3+\ldots}=\cos \left(\theta_{2}+\theta_{3}+\ldots\right)$, $\mathrm{s}_{2+3+\ldots}=\sin \left(\theta_{2}+\theta_{3}+\ldots\right), \mathrm{y}$

$\delta_{1}=c_{\varphi} s_{2+3+4} p_{z}+c_{\varphi} s_{2+3+4} s_{5}+c_{\varphi} c_{2+3} a_{34}+c_{\varphi} c_{2} a_{23}$

donde $\mathrm{p}_{z}$ es la distancia de la herramienta hasta el quinto elemento, $\mathrm{p}_{\mathrm{z}}=102 \mathrm{~mm}$.

$$
\delta_{2}=-s_{\varphi} s_{2+3+4} p_{z}+s_{\varphi} s_{2+3+4} s_{5}+s_{\varphi} c_{2+3} a_{34}+s_{\varphi} c_{2} a_{23}
$$

$$
\delta_{3}=-c_{2+3+4} p_{z}-c_{2+3+4} S_{5}+s_{2+3} a_{34}+s_{2} a_{23} .
$$

TABLA I

Parámetros del Manipulador Movemaster RV-M1

\begin{tabular}{|c|c|}
\hline $\begin{array}{c}\text { Distancia de la articulación } \\
{[\mathbf{m m}]}\end{array}$ & $\begin{array}{c}\text { Distancia del elemento } \\
{[\mathbf{m m}]}\end{array}$ \\
\hline-- & $\mathrm{a}_{12}=0$ \\
\hline $\mathrm{S}_{2}=0$ & $\mathrm{a}_{23}=250$ \\
\hline $\mathrm{S}_{3}=0$ & $\mathrm{a}_{34}=160$ \\
\hline $\mathrm{S}_{4}=0$ & $\mathrm{a}_{45}=0$ \\
\hline $\mathrm{S}_{5}=72$ & $\mathrm{a}_{56}=0$ \\
\hline $\begin{array}{c}\text { Ángulo de elemento } \\
\left.{ }^{\circ}\right]\end{array}$ & Ángulo de articulación \\
{$\left[{ }^{\circ}\right]$}
\end{tabular}

*: Variable, hallada en la cinemática inversa

Fuente. Elaboración propia, datos tomados de [15].

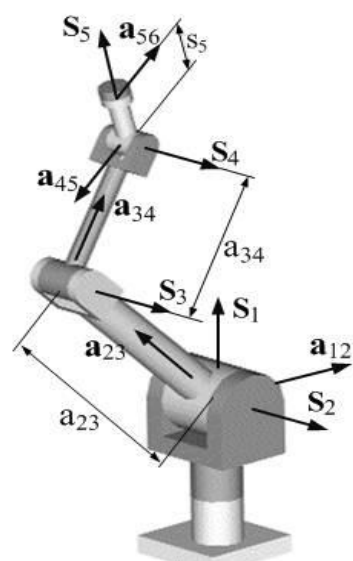

(a) Distancia de junta y de elemento

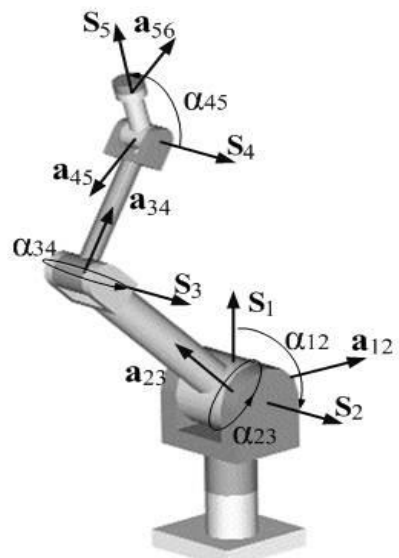

(c) Ángulos de elementos

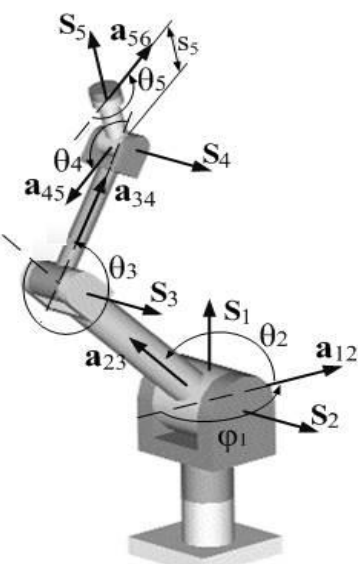

(b) Ángulos de junta

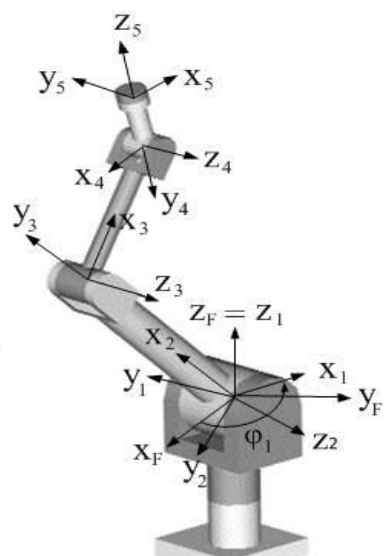

(d) Ejes coordenados
Figura 2. Parámetros cinemáticos unificados para la representación del RV-M1 (a) distancias (b) ángulos de junta (c) ángulos de elementos (d) ejes coordenados. Fuente. Elaboración propia. 


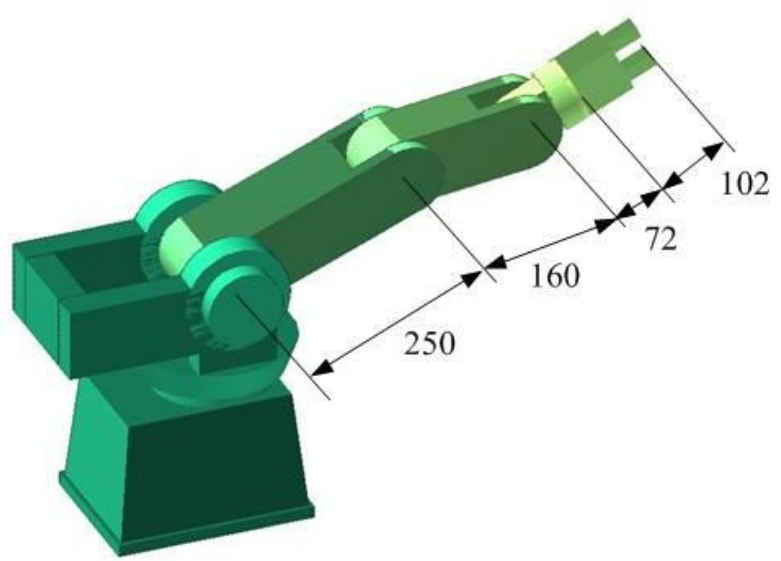

Figura 3. Dimensiones principales del robot Mitsubishi Movemaster RV-M1. Fuente. Elaboración propia con datos de [15].

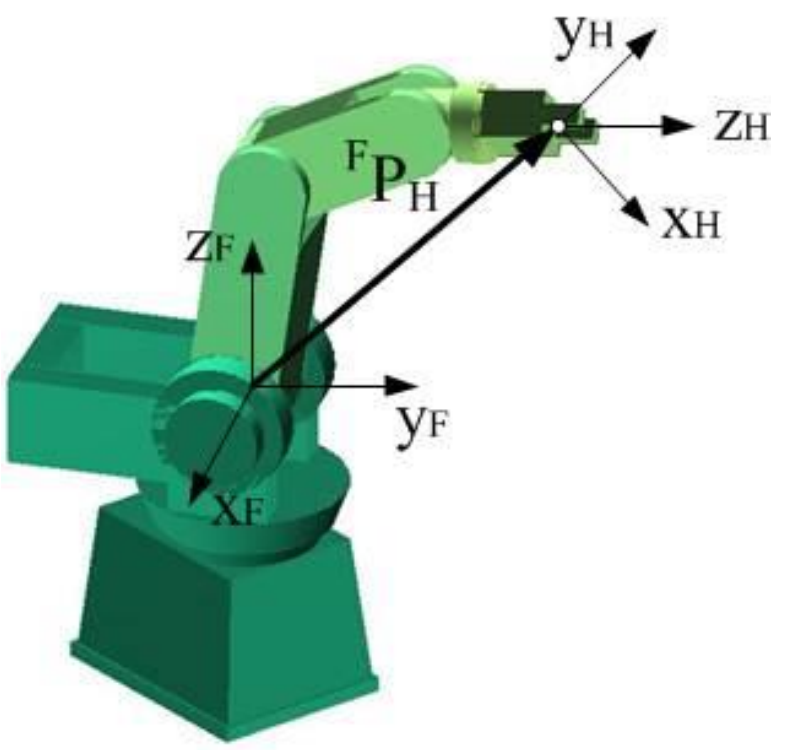

Figura 4. Posición ${ }^{\mathrm{F}} \mathrm{P}_{\mathrm{H}} \mathrm{y}$ orientación de la herramienta $\left\{\mathrm{x}_{\mathrm{H}-\mathrm{yH}^{-}}\right.$ $\left.\mathrm{Z}_{\mathrm{H}}\right\}$ de la herramienta en el marco fijo $\left\{\mathrm{x}_{\mathrm{F}}-\mathrm{yF}_{\mathrm{F}}-\mathrm{ZF}\right\}$. Fuente. Elaboración propia.

2.1.1 Cinemática directa de posición. La cinemática directa, es decir, hallar la posición y orientación de la herramienta a partir de los ángulos de junta conocidos y las distancias de junta y ángulos, se resuelve de la siguiente manera. El vector posición de la herramienta, ${ }^{\mathrm{F}} \mathbf{P}_{\mathrm{H}}$, es extraído de los elementos $(1,4),(2,4)$ y $(3,4)$ de la matriz del lado derecho de la Ecuación (4), para las componentes $\mathrm{x}$, y e $\mathrm{z}$, respectivamente, expresado mediante la Ecuación (10).

$$
\mathbf{P}_{\mathrm{F}}=\left[\begin{array}{l}
\delta_{1} \\
\delta_{2} \\
\delta_{3}
\end{array}\right]
$$

La orientación de la herramienta es obtenida a partir de los nueve elementos $(1,1)$ a $(3,3)$ de la matriz de rotación, ${ }_{\mathrm{j}}^{\mathrm{i}} \mathrm{R}$, extraída de la Ecuación 4, para expresar los ángulos del navegante conocidos como Roll-Pitch-Yaw (Guiñada-Cabeceo-Alabeo) [16], a partir de la ecuación 11 para el ángulo de guiñada $\alpha$, ecuación 12 para el ángulo de cabeceo $\beta$ y ecuación 13 para el ángulo de alabeo, $\gamma$, los cuales son los ángulos que la herramienta ha rotado con respecto a los ejes del marco fijo $\mathrm{x}$, y e $\mathrm{z}$, respectivamente, donde $\mathrm{r}_{\mathrm{hk}}$ denota la componente de la matriz de rotación.

$$
\begin{aligned}
& \alpha=\operatorname{atan}\left(\frac{r_{32}}{r_{33}}\right) \\
& \beta=-\operatorname{asin}\left(r_{31}\right) \\
& \gamma=\operatorname{atan}\left(\frac{r_{21}}{r_{11}}\right)
\end{aligned}
$$

2.1.1 Cinemática inversa de posición. En la cinemática inversa se deben hallar los ángulos de posición de los elementos móviles del robot, conocidos los valores de la posición y orientación de la herramienta en el marco fijo. Es decir, se deben hallar los ángulos $\varphi_{1}, \theta_{2}, \theta_{3}, \theta_{4}$ y $\theta_{5}$, a partir de los elementos de la matriz en el lado derecho de la ecuación 4. En este trabajo se adoptó del método matricial expuesto en [17] para el robot RV-M1, el cual puede ser consultado para un detallado proceso de solución. Mediante el método de sucesivas premultiplicaciones de la ecuación 3 , en [17] se demostraron las expresiones para los ángulos $\varphi_{1}, \theta_{2}, \theta_{3}, \theta_{4}$ y $\theta_{5}$, presentadas en las ecuaciones 14 a 18 , respectivamente.

$$
\begin{gathered}
\varphi_{1}=\operatorname{atan} 2\left(\mathrm{r}_{23}, \mathrm{r}_{13}\right) \\
\theta_{2}=\operatorname{atan} 2\left(\frac{\mathrm{Q}}{\sqrt{\mathrm{P}^{2}+\mathrm{Q}^{2}}}, \frac{\mathrm{P}}{\sqrt{\mathrm{P}^{2}+\mathrm{Q}^{2}}}\right) \pm \cos ^{-1}\left(\frac{-\mathrm{R}}{\sqrt{\mathrm{P}^{2}+\mathrm{Q}^{2}}}\right),
\end{gathered}
$$

donde: $\mathrm{P}=\left(\mathrm{S}_{5}+\mathrm{p}_{\mathrm{z}}\right)\left(\mathrm{r}_{13} \mathrm{c}_{\varphi}+\mathrm{r}_{23} \mathrm{~s}_{\varphi}\right)-\left(\mathrm{r}_{14} \mathrm{c}_{\varphi}+\mathrm{r}_{24} \mathrm{~s}_{\varphi}\right)$, $\mathrm{Q}=\left(\mathrm{S}_{5}+\mathrm{p}_{\mathrm{z}}\right) \mathrm{r}_{33}-\mathrm{r}_{34}, \mathrm{R}=\mathrm{a}_{23}+\mathrm{a}_{34} \mathrm{c}_{3}$.

$\theta_{3}=$

$\operatorname{atan} 2\left(\frac{\mathrm{r}_{34}+\left(\mathrm{S}_{5}+\mathrm{p}_{\mathrm{z}}\right) \mathrm{c}_{2+3+4}-\mathrm{a}_{23} \mathrm{~s}_{2-} \mathrm{a}_{34} \mathrm{~s}_{2} \mathrm{c}_{3}}{\mathrm{a}_{34} \mathrm{c}_{2}}, \frac{\mathrm{A}^{2}+\mathrm{B}^{2}-\mathrm{a}_{23}^{2}-\mathrm{a}_{34}^{2}}{2 \mathrm{a}_{23} \mathrm{a}_{34}}\right)$

donde:

$$
\theta_{4}=\operatorname{atan} 2\left(s_{4}, c_{4}\right),
$$

$$
\begin{gathered}
\mathrm{s}_{4}=\frac{\mathrm{c}_{2+3}\left(\mathrm{r}_{14} \mathrm{c}_{\varphi}+\mathrm{r}_{24} \mathrm{~s}_{\varphi}\right)+\mathrm{r}_{34} \mathrm{~s}_{2+3}-\mathrm{a}_{23} \mathrm{c}_{3}-\mathrm{a}_{34}}{\mathrm{~s}_{5}+\mathrm{p}_{\mathrm{z}}}, \\
\mathrm{c}_{4}=\frac{\mathrm{s}_{2+3}\left(\mathrm{r}_{14} \mathrm{c}_{\varphi}+\mathrm{r}_{24} \mathrm{~s}_{\varphi}\right)-\mathrm{r}_{34} \mathrm{c}_{2+3}-\mathrm{a}_{23} \mathrm{~s}_{3}}{\mathrm{~S}_{5}+\mathrm{p}_{\mathrm{z}}} \\
\theta_{5}=\operatorname{atan} 2\left(\mathrm{r}_{11} \mathrm{~s}_{\varphi}-\mathrm{r}_{21} \mathrm{c}_{\varphi}, \mathrm{r}_{12} \mathrm{~s}_{\varphi}-\mathrm{r}_{22} \mathrm{c}_{\varphi}\right) .
\end{gathered}
$$




\subsection{Dinámica}

El modelo dinámico del RV-M1 fue desarrollado usando el método energético de Euler-Lagrange, sugerido para manipuladores seriales, por cuanto no se requiere encontrar las fuierzas de restricción $[16 ; 18]$. El Lagrangiano $\mathrm{L}$ dado por:

$$
\mathrm{L}=\frac{1}{2} \dot{\mathrm{q}}^{\mathrm{T}} \mathrm{M} \dot{\mathrm{q}}+\sum_{\mathrm{i}=1}^{\mathrm{n}} \mathrm{m}_{\mathrm{i}} \mathrm{g}^{\mathrm{T}} \mathrm{P}_{\mathrm{ci}},
$$

donde $\mathrm{M}$ es la matriz de masa del manipulador (tamaño $5 \times 5$ en este caso), $\dot{q}$ es el vector de razón de cambio de las coordenadas generalizadas dado por

$$
\dot{\mathrm{q}}=\left[\begin{array}{c}
\dot{\varphi}_{1} \\
\dot{\theta}_{2} \\
\dot{\theta}_{3} \\
\dot{\theta}_{4} \\
\dot{\theta}_{5}
\end{array}\right],
$$

$\mathrm{n}$ es el número de coordenadas generalizadas $\mathrm{n}=5, \mathrm{~m}_{\mathrm{i}}$ es la masa del elemento $i, g$ es el vector gravedad dado por la ecuación 21 y $\mathrm{P}_{\mathrm{Ci}}$ es el vector del centro de masa del elemento i, tomado de las posiciones $(1,4),(2,4)$ y $(3,4)$ de la matriz de transformación ${ }_{\mathrm{i}}^{\mathrm{F}} \mathrm{T}$ del centro de masa del elemento $i$, desplazado una distancia $b_{i}$ a lo largo de la dirección del vector del elemento, desde el origen del marco de referencia local de cada elemento, expresadas en el marco fijo por las ecuaciones 22 a 26.

$$
\begin{gathered}
\mathrm{g}=\left[\begin{array}{c}
0 \\
0 \\
-9.81
\end{array}\right] \frac{\mathrm{m}}{\mathrm{s}^{2}} \\
{ }_{1} \mathrm{~T}=\left[\begin{array}{cccc}
\mathrm{c}_{\varphi} & -\mathrm{s}_{\varphi} & 0 & -\mathrm{b}_{1} \mathrm{c}_{\varphi} \\
\mathrm{s}_{\varphi} & \mathrm{c}_{\varphi} & 0 & -\mathrm{b}_{1} \mathrm{~s}_{\varphi} \\
0 & 0 & 1 & 0 \\
0 & 0 & 0 & 1
\end{array}\right],
\end{gathered}
$$

donde $\mathrm{c}_{\varphi}=\cos \left(\varphi_{1}\right), \mathrm{s}_{\varphi}=\sin \left(\varphi_{1}\right)$.

$$
\begin{aligned}
{ }_{2} \mathrm{~T} & =\left[\begin{array}{cccc}
\mathrm{c}_{\varphi} \mathrm{c}_{2} & -\mathrm{c}_{\varphi} \mathrm{s}_{2} & \mathrm{~s} \varphi & \mathrm{c}_{\varphi} \mathrm{c}_{2} \mathrm{~b}_{2} \\
\mathrm{~s}_{\varphi} \mathrm{c}_{2} & -\mathrm{s}_{\varphi} \mathrm{s}_{2} & -\mathrm{c}_{\varphi} & \mathrm{s}_{\varphi} \mathrm{c}_{2} \mathrm{~b}_{2} \\
\mathrm{~s}_{2} & \mathrm{c}_{2} & 0 & \mathrm{~s}_{2} \mathrm{~b}_{2} \\
0 & 0 & 0 & 1
\end{array}\right], \\
{ }_{3} \mathrm{~T} & =\left[\begin{array}{cccc}
\mathrm{c}_{\varphi} \mathrm{c}_{2+3} & -\mathrm{c}_{\varphi} \mathrm{s}_{2+3} & \mathrm{~s}_{\varphi} & \mathrm{c}_{\varphi} \mathrm{A}_{1} \\
\mathrm{~s}_{\varphi} \mathrm{c}_{2+3} & -\mathrm{s}_{\varphi} \mathrm{s}_{2+3} & -\mathrm{c}_{\varphi} & \mathrm{s}_{\varphi} \mathrm{A}_{1} \\
\mathrm{~s}_{2+3} & \mathrm{c}_{2+3} & 0 & \mathrm{~A}_{2} \\
0 & 0 & 0 & 1
\end{array}\right],
\end{aligned}
$$

donde $c_{2+3+\cdots}=\cos \left(\theta_{2}+\theta_{3}+\cdots\right)$, $\mathrm{s}_{2+3+\cdots}=\sin \left(\theta_{2}+\theta_{3}+\cdots\right)$, $A_{1}=c_{2} a_{23}+c_{2+3} b_{3}, A_{2}=s_{2} a_{23}+b_{3} s_{2+3}$.

$$
{ }_{4}^{\mathrm{F}} \mathrm{T}=\left[\begin{array}{cccc}
\mathrm{c}_{\varphi} \mathrm{c}_{2+3+4} & -\mathrm{c}_{\varphi} \mathrm{s}_{2+3+4} & \mathrm{~s}_{\varphi} & \mathrm{A}_{3} \\
\mathrm{~s}_{\varphi} \mathrm{c}_{2+3+4} & -\mathrm{s}_{\varphi} \mathrm{s}_{2+3+4} & -\mathrm{c}_{\varphi} & \mathrm{A}_{4} \\
\mathrm{~s}_{2+3+4} & \mathrm{c}_{2+3+4} & 0 & \mathrm{~A}_{5} \\
0 & 0 & 0 & 1
\end{array}\right]
$$

donde $\mathrm{A}_{3}=\mathrm{c}_{\varphi}\left(\mathrm{c}_{2} \mathrm{a}_{23}+\mathrm{c}_{2+3} \mathrm{a}_{34}+\mathrm{s}_{2+3+4} \mathrm{~b}_{4}\right)$,

$\mathrm{A}_{4}=\mathrm{s}_{\varphi}\left(\mathrm{c}_{2} \mathrm{a}_{23}+\mathrm{c}_{2+3} \mathrm{a}_{34}+\mathrm{s}_{2+3+4} \mathrm{~b}_{4}\right)$,

$A_{5}=s_{2} a_{23}+s_{2+3} a_{34}-s_{2+3+4} b_{4}$.

$$
{ }_{5}^{\mathrm{F}} \mathrm{T}=\left[\begin{array}{cccc}
\mathrm{s}_{\varphi} \mathrm{s}_{5}+\mathrm{A}_{6} \mathrm{C}_{5} & \mathrm{~s}_{\varphi} \mathrm{c}_{5}-\mathrm{A}_{6} \mathrm{~s}_{5} & \mathrm{~A}_{8} & \mathrm{~A}_{11} \\
-\mathrm{c}_{\varphi} \mathrm{s}_{5}+\mathrm{A}_{7} \mathrm{c}_{5} & -\mathrm{c}_{\varphi} \mathrm{c}_{5}-\mathrm{A}_{7} \mathrm{~s}_{5} & \mathrm{~A}_{9} & \mathrm{~A}_{12} \\
\mathrm{~s}_{2+3+4} \mathrm{C}_{5} & -\mathrm{s}_{2+3+4} \mathrm{~s}_{5} & \mathrm{~A}_{10} & \mathrm{~A}_{13} \\
0 & 0 & 0 & 1
\end{array}\right],
$$

donde $\mathrm{A}_{6}=\mathrm{c}_{\varphi} \mathrm{c}_{2+3+4}, \mathrm{~A}_{7}=\mathrm{s}_{\varphi} \mathrm{c}_{2+3+4}$,

$\mathrm{A}_{8}=\mathrm{c}_{\varphi} \mathrm{s}_{2+3+4}, \mathrm{~A}_{9}=\mathrm{s}_{\varphi} \mathrm{s}_{2+3+4}, \mathrm{~A}_{10}=-\mathrm{c}_{2+3+4}, \mathrm{~A}_{11}=$

$\mathrm{c}_{\varphi}\left(\mathrm{c}_{2} \mathrm{a}_{23}+\mathrm{c}_{2+3} \mathrm{a}_{34}+\mathrm{s}_{2+3+4}\left(\mathrm{~S}_{5}+\mathrm{b}_{5}\right)\right)$,

$A_{12}=s_{\varphi}\left(c_{2} a_{23}+c_{2+3} a_{34}+s_{2+3+4}\left(S_{5}+b_{5}\right)\right)$,

$A_{13}=s_{2} a_{23}+s_{2+3} a_{34}-c_{2+3+4}\left(S_{5}+b_{5}\right)$.

Para facilitar la obtención de las derivadas de la ecuación 19, se expande el término de la energía cinética $\left(\frac{1}{2} \dot{\mathrm{q}}^{\mathrm{T}} \mathrm{M} \dot{\mathrm{q}}\right)$ como una suma escalar dada en la ecuación 27.

$$
\mathrm{L}=\left(\frac{1}{2} \sum_{\mathrm{i}=1}^{\mathrm{n}} \sum_{\mathrm{j}=1}^{\mathrm{n}} \mathrm{M}_{\mathrm{ij}} \dot{\mathrm{q}}_{\mathrm{i}} \dot{\mathrm{q}}_{\mathrm{j}}\right)+\sum_{\mathrm{i}=1}^{\mathrm{n}}\left(\mathrm{m}_{\mathrm{i}} \mathrm{g}^{\mathrm{T}} \mathrm{P}_{\mathrm{ci}}\right)
$$

Tomando las derivadas de $\mathrm{q}_{i}\left(\mathrm{q}_{\mathrm{i}}\right.$ son las componentes del vector de coordenadas generalizadas, $\mathrm{q}$, dado por la ecuación 28) y $\dot{\mathrm{q}}_{\mathrm{i}}$ con respecto al tiempo, t, del Lagrangiano en la ecuación 27 se obtiene la ecuación 29 , donde $\tau_{\mathrm{i}}$ es la fuerza o momento externo actuando sobre el elemento i, $\mathrm{C}_{\mathrm{i}}$ es el vector de acople de velocidad de cada elemento dado por la ecuación 30 con $\mathrm{G}_{\mathrm{i}}$ como el vector gravitacional dado por la ecuación 31 .

$$
\begin{gathered}
q=\left[\begin{array}{c}
\varphi_{1} \\
\theta_{2} \\
\theta_{3} \\
\theta_{4} \\
\theta_{5}
\end{array}\right] \quad \\
\tau_{i}=\sum_{j=1}^{n} M_{i j} \ddot{q}_{j}+C_{i}+G_{i} \\
C_{i}=\sum_{j=1}^{n} \sum_{k=1}^{n}\left[\frac{\partial M_{i j}}{\partial q_{k}}-\frac{1}{2} \frac{\partial M_{j k}}{\partial q_{i}} \dot{\mathrm{q}}_{j} \dot{q}_{k}\right] \\
G_{i}=-\sum_{j=1}^{n}\left(m_{j} g^{T} J_{v j}^{i}\right)
\end{gathered}
$$

La fila i-ésima de los Jacobianos de velocidad del elemento $\mathrm{j}, \mathrm{J}_{\mathrm{vj}}^{\mathrm{i}}$, está dada por la respectiva columna de las filas 1 a 3 de la matriz Jacobiano del centro de masa de cada elemento en el marco fijo, $\mathrm{J}_{\mathrm{CM} 1}$ a $\mathrm{J}_{\mathrm{CM} 5}$, generados a 
partir de las matrices de transformación presentadas en las ecuaciones 22 a 26, generando las ecuaciones 32 a 36 .

$$
\begin{aligned}
\mathrm{J}_{\mathrm{CM} 1} & =\left[\begin{array}{ccccc}
\mathrm{s}_{\varphi} \mathrm{b}_{1} & 0 & 0 & 0 & 0 \\
-\mathrm{c}_{\varphi} \mathrm{b}_{1} & 0 & 0 & 0 & 0 \\
0 & 0 & 0 & 0 & 0 \\
0 & 0 & 0 & 0 & 0 \\
0 & 0 & 0 & 0 & 0 \\
1 & 0 & 0 & 0 & 0
\end{array}\right] \\
\mathrm{J}_{\mathrm{CM} 2} & =\left[\begin{array}{ccccc}
-\mathrm{s}_{\varphi} \mathrm{c}_{2} \mathrm{~b}_{2} & -\mathrm{c}_{\varphi} \mathrm{s}_{2} \mathrm{~b}_{2} & 0 & 0 & 0 \\
\mathrm{c}_{\varphi} \mathrm{c}_{2} \mathrm{~b}_{2} & -\mathrm{s}_{\varphi} \mathrm{s}_{2} \mathrm{~b}_{2} & 0 & 0 & 0 \\
0 & \mathrm{c}_{2} \mathrm{~b}_{2} & 0 & 0 & 0 \\
0 & \mathrm{~s}_{\varphi} & 0 & 0 & 0 \\
0 & -\mathrm{c}_{\varphi} & 0 & 0 & 0 \\
1 & 0 & 0 & 0 & 0
\end{array}\right] \\
\mathrm{J}_{\mathrm{CM} 3} & {\left[\begin{array}{ccccc}
-\mathrm{s}_{\varphi} \mathrm{A}_{14} & -\mathrm{c}_{\varphi} \mathrm{A}_{15} & -\mathrm{A}_{16} & 0 & 0 \\
\mathrm{c}_{\varphi} \mathrm{A}_{14} & -\mathrm{s}_{\varphi} \mathrm{A}_{15} & -\mathrm{A}_{17} & 0 & 0 \\
0 & \mathrm{~A}_{14} & \mathrm{~A}_{18} & 0 & 0 \\
0 & \mathrm{~s}_{\varphi} & \mathrm{s}_{\varphi} & 0 & 0 \\
0 & -\mathrm{c}_{\varphi} & -\mathrm{c}_{\varphi} & 0 & 0 \\
1 & 0 & 0 & 0 & 0
\end{array}\right], }
\end{aligned}
$$

donde $A_{14}=c_{2} a_{23}+c_{2+3} b_{3}$,

$A_{15}=s_{2} a_{23}+s_{2+3} b_{3}, A_{16}=c_{\varphi}\left(s_{2+3} b_{3}\right)$,

$A_{17}=s_{\varphi}\left(s_{2+3} b_{3}\right), A_{18}=c_{2+3} b_{3}$.

$$
\mathrm{J}_{\mathrm{CM} 4}=\left[\begin{array}{ccccc}
-\mathrm{A}_{19} & -\mathrm{A}_{21} & -\mathrm{A}_{24} & \mathrm{~A}_{27} & 0 \\
\mathrm{~A}_{20} & -\mathrm{A}_{22} & -\mathrm{A}_{25} & \mathrm{~A}_{28} & 0 \\
0 & \mathrm{~A}_{23} & \mathrm{~A}_{26} & \mathrm{~A}_{29} & 0 \\
0 & \mathrm{~S}_{\varphi} & \mathrm{S}_{\varphi} & \mathrm{S}_{\varphi} & 0 \\
0 & -\mathrm{c}_{\varphi} & -\mathrm{c}_{\varphi} & -\mathrm{c}_{\varphi} & 0 \\
1 & 0 & 0 & 0 & 0
\end{array}\right]
$$

donde $\mathrm{A}_{19}=\mathrm{s}_{\varphi}\left(\mathrm{c}_{2} \mathrm{a}_{23}+\mathrm{c}_{2+3} \mathrm{a}_{34}+\mathrm{s}_{2+3+4} \mathrm{~b}_{4}\right), \mathrm{A}_{20}=$ $\mathrm{c}_{\varphi}\left(\mathrm{c}_{2} \mathrm{a}_{23}+\mathrm{c}_{2+3} \mathrm{a}_{34}+\mathrm{s}_{2+3+4} \mathrm{~b}_{4}\right)$,

$\mathrm{A}_{21}=\mathrm{c}_{\varphi}\left(\mathrm{s}_{2} \mathrm{a}_{23}+\mathrm{s}_{2+3} \mathrm{a}_{34}-\mathrm{c}_{2+3+4} \mathrm{~b}_{4}\right)$,

$A_{22}=s_{\varphi}\left(s_{2} a_{23} s_{2+3} a_{34}-c_{2+3+4} b_{4}\right)$,

$A_{23}=c_{2} a_{23}+c_{2+3} a_{34}+c_{2+3+4} b_{4}$,

$\mathrm{A}_{24}=\mathrm{c}_{\varphi}\left(\mathrm{s}_{2+3} \mathrm{a}_{34}-\mathrm{c}_{2+3+4} \mathrm{~b}_{4}\right)$,

$A_{25}=s_{\varphi}\left(s_{2+3} a_{34}-c_{2+3+4} b_{4}\right)$,

$\mathrm{A}_{26}=\mathrm{c}_{2+3} \mathrm{a}_{34}+\mathrm{s}_{2+3+4} \mathrm{~b}_{4}, \mathrm{~A}_{27}=\mathrm{c}_{\varphi} \mathrm{c}_{2+3+4} \mathrm{~b}_{4}$,

$A_{28}=s_{\varphi} c_{2+3+4} b_{4}, A_{29}=s_{2+3+4} b_{4}$.

$$
\mathrm{J}_{\mathrm{CM} 5}=\left[\begin{array}{ccccc}
-\mathrm{A}_{20} & -\mathrm{A}_{22} & \mathrm{~A}_{25} & \mathrm{~A}_{28} & 0 \\
\mathrm{~A}_{21} & -\mathrm{A}_{23} & \mathrm{~A}_{26} & \mathrm{~A}_{29} & 0 \\
0 & \mathrm{~A}_{24} & \mathrm{~A}_{27} & \mathrm{~A}_{30} & 0 \\
0 & \mathrm{~S}_{\varphi} & \mathrm{S}_{\varphi} & \mathrm{S}_{\varphi} & \mathrm{A}_{31} \\
0 & -\mathrm{c}_{\varphi} & -\mathrm{c}_{\varphi} & -\mathrm{c}_{\varphi} & \mathrm{A}_{32} \\
1 & 0 & 0 & 0 & -\mathrm{A}_{33}
\end{array}\right] \text {, }
$$

donde

$$
\begin{aligned}
& A_{20}=s_{\varphi}\left(c_{2} a_{23}+c_{2+3} a_{34}+s_{2+3+4}\left(b_{5}+s_{5}\right)\right), \\
& A_{21}=c_{\varphi}\left(c_{2} a_{23}+c_{2+3} a_{34}+s_{2+3+4}\left(b_{5}+s_{5}\right)\right), \\
& A_{22}=c_{\varphi}\left(s_{2} a_{23}+s_{2+3} a_{34}-s_{2+3+4}\left(b_{5}+S_{5}\right)\right), \\
& A_{23}=s_{\varphi}\left(s_{2} a_{23}+s_{2+3} a_{34}-c_{2+3+4}\left(b_{5}+s_{5}\right)\right), \\
& A_{24}=c_{2} a_{23}+c_{2+3} a_{34}+s_{2+3+4}\left(b_{5}+s_{5}\right), \\
& A_{25}=c_{\varphi}\left(c_{2+3} S_{5}-s_{2+3} a_{23}+c_{2+3+4} b_{5}\right), \\
& A_{26}=s_{\varphi}\left(c_{2+3} S_{5}-s_{2+3} a_{23}+c_{2+3+4} b_{5}\right), \\
& A_{27}=c_{2+3} S_{5}+s_{2+3} a_{34}+c_{2+3+4} b_{5}, \\
& A_{28}=c_{\varphi} c_{2+3+4}\left(S_{5}+b_{5}\right), \\
& A_{29}=s_{\varphi} c_{2+3+4}\left(S_{5}+b_{5}\right), \\
& A_{30}=s_{2+3+4}\left(S_{5}+b_{5}\right), A_{31}=c_{\varphi} s_{2+3+4}, \\
& A_{32}=s_{\varphi} s_{2+3+4}, A_{33}=c_{2+3+4} .
\end{aligned}
$$

Se han obtenido expresiones en el lado derecho de la ecuación 37, donde $\mathrm{M}$ es la matriz de inercia, $\mathrm{C}$ es el vector de fuerza centrífuga y de Coriolis, $G$ es el vector de pares gravitacionales. El término $\tau$ es la fuerza generalizada de momentos y cargas en las juntas. Se ha despreciado la fricción viscosa en las articulaciones. Los términos q, d y ä son vectores que representan la posición (ver ecuación 28), velocidad (ver ecuación 20) y aceleración (ver ecuación 38) articular del robot, respectivamente.

$$
\tau=M(q) \ddot{q}+C(q, \dot{q}) \dot{q}+G(q)
$$

$$
\ddot{\mathrm{q}}=\left[\begin{array}{c}
\ddot{\varphi}_{1} \\
\ddot{\theta}_{2} \\
\ddot{\theta}_{3} \\
\ddot{\theta}_{4} \\
\ddot{\theta}_{5}
\end{array}\right]
$$

\subsection{Determinación de los parámetros dinámicos}

El conocimiento de cada término en la ecuación 37 implica la expansión de la matriz de masa $\mathrm{M}$ dada por

$$
\mathrm{M}=\left[\sum_{\mathrm{i}=1}^{\mathrm{n}}\left(\mathrm{J}_{\mathrm{vi}}^{\mathrm{T}} \mathrm{m}_{\mathrm{i}} \mathrm{J}_{v i}+\frac{1}{2} \mathrm{~J}_{\omega \mathrm{i}}^{\mathrm{T}} \mathrm{I}_{\mathrm{i}} \mathrm{J}_{\omega \mathrm{i}}\right)\right],
$$

donde $\mathrm{J}_{\mathrm{vi}}$ es el Jacobiano de velocidad del centro de masa del elemento i, dado por las primeras tres filas de la matriz Jacobiano del elemento, presentado en las ecuaciones 32 a 36; $\mathrm{J}_{\omega \mathrm{i}}$ es el Jacobiano de velocidad angular del elemento i-ésimo, tomado de las últimas tres filas de la matriz Jacobiano del respectivo elemento, ecuaciones 32 a 36 ; e $I_{i}$ es la matriz de inercia del elemento i-ésimo en el marco fijo, la cual se relaciona con la matriz de inercia del elemento en su propio marco de referencia, a través de la expresión:

$$
\mathrm{I}_{\mathrm{i}}={ }^{\mathrm{F}} \mathrm{R}_{\mathrm{i}}{ }^{\mathrm{i}} \mathrm{I}_{\mathrm{i}}{ }^{\mathrm{F}} \mathrm{R}_{\mathrm{i}}^{\mathrm{T}}
$$


donde ${ }^{\mathrm{F}} \mathrm{R}_{\mathrm{i}}$ es la matriz de rotación del elemento i-ésimo en el marco fijo, la cual es tomada de los elementos $(1,1)$ al $(3,3)$ de la matriz de transformación respectiva de las ecuaciones 22 a 26. Los valores de masa, $\mathrm{m}_{\mathrm{i}}$, para el modelo dinámico se toman directamente de la información proporcionada por [15]: $\mathrm{m}_{1}=4.584 \mathrm{~kg}, \mathrm{~m}_{2}=$ $4.961 \mathrm{~kg}, \mathrm{~m}_{3}=2.172 \mathrm{~kg}, \mathrm{~m}_{4}=1.053 \mathrm{~kg}, \mathrm{~m}_{5}=0.6 \mathrm{~kg}$. En lo que respecta a la inercia, un modelamiento de cada elemento del robot podría ser un paralelepípedo de masa $\mathrm{m}$ como el mostrado en la figura 5, con matriz de inercia en su marco de referencia dada por la ecuación 41, donde las dimensiones $\mathrm{w}, \mathrm{h}$ y a son definidas en la figura 5.

$$
I_{i}=\left[\begin{array}{ccc}
\frac{\mathrm{m}\left(\mathrm{w}^{2}+\mathrm{h}^{2}\right)}{12} & 0 & 0 \\
0 & \frac{\mathrm{m}\left(\mathrm{h}^{2}+\mathrm{a}^{2}\right)}{12} & 0 \\
0 & 0 & \frac{\mathrm{m}\left(\mathrm{a}^{2}+\mathrm{w}^{2}\right)}{12}
\end{array}\right] \text {, }
$$

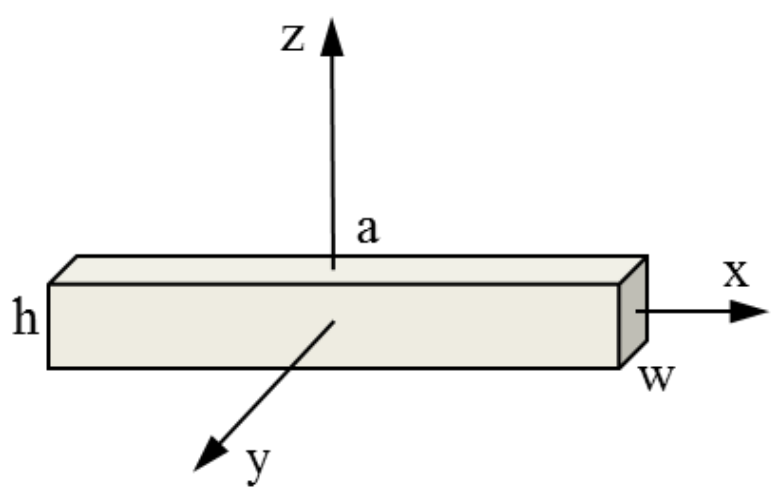

Figura 5. Paralelepípedo y ejes centroidales. Fuente. Elaboración propia.

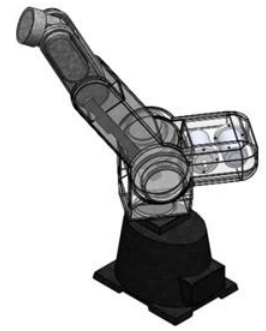

(a) Vista isométrica del RV-M1

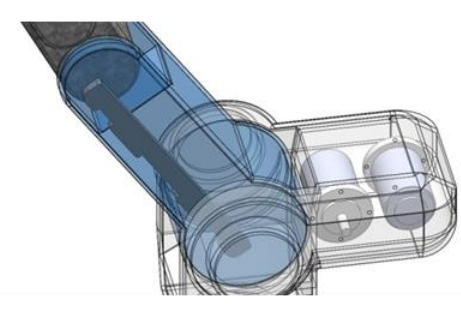

(b) Acercamiento de isométrica del RV-M1
Figura 6. Componentes internos del robot (a) feneral (b) acercamiento. Fuente. Elaboración propia.

Sin embargo, puesto que los eslabones del robot son piezas huecas con componentes internos, tratando de obtener valores más confiables de inercia, se generó un modelo $\mathrm{CAD}$ en SolidWorks para cada elemento teniendo en cuenta sus componentes: servomotores, bases, poleas y cables entre otros (ver figura 6), asignando el material y masa correspondiente a cada elemento.

A continuación se utilizó la herramienta de SolidWorks "Calcular propiedades físicas" para obtener las propiedades de inercia en el marco centroidal de cada elemento. En la figura 7 se ilustran los resultados generados para el antebrazo (elemento 2), por citar un ejemplo. Las propiedades de todos los elementos son mostradas en la tabla II y estos valores son remplazados en la correspondiente matriz de inercia del elemento mostrada en la ecuación 42, donde $\mathrm{I}_{\mathrm{ij}}$ denota el momento de inercia que relaciona los ejes i e $\mathrm{j}$.

$$
I_{i}=\left[\begin{array}{lll}
I_{x x} & I_{x y} & I_{x z} \\
I_{y x} & I_{y y} & I_{y z} \\
I_{z x} & I_{z y} & I_{z z}
\end{array}\right]
$$

TABLA II

INERCIA DE LOS ELEMENTOS CON RESPECTO A SUS EJES DE

\begin{tabular}{|c|c|c|}
\hline \multicolumn{3}{|c|}{ Inercia Hombro $\left[\mathrm{kg}-\mathrm{m}^{2}\right]$} \\
\hline $\mathrm{I}_{\mathrm{xx}}=0,08250219$ & $I_{x y}=-3 e-8$ & $\mathrm{I}_{\mathrm{xz}}=1,8409 \mathrm{e}-4$ \\
\hline$I_{y x}=-3 e-8$ & $\mathrm{I}_{\mathrm{yy}}=0,08460189$ & $\mathrm{I}_{\mathrm{yz}}=-5,459 \mathrm{e}-5$ \\
\hline $\mathrm{I}_{\mathrm{zx}}=1,8409 \mathrm{e}-4$ & $\mathrm{I}_{\mathrm{zy}}=-5,459 \mathrm{e}-5$ & $\mathrm{I}_{\mathrm{zz}}=0,01109258$ \\
\hline \multicolumn{3}{|c|}{ Inercia Antebrazo $\left[\mathrm{kg}-\mathrm{m}^{2}\right]$} \\
\hline $\mathrm{I}_{\mathrm{xx}}=0,07876122$ & $\mathrm{I}_{\mathrm{xy}}=-3,3315 \mathrm{e}-4$ & $\mathrm{I}_{\mathrm{xz}}=-1,69624 \mathrm{e}-3$ \\
\hline$I_{y x}=-3,3315 e-4$ & $\mathrm{I}_{\mathrm{yy}}=0,07933805$ & $\mathrm{I}_{\mathrm{yz}}=-8,7074 \mathrm{e}-4$ \\
\hline$I_{z x}=-1,69624 e-3$ & $\mathrm{I}_{\mathrm{zy}}=-8,7074 \mathrm{e}-4$ & $\mathrm{I}_{\mathrm{zz}}=0,01213611$ \\
\hline \multicolumn{3}{|c|}{ Inercia Brazo $\left[\mathrm{kg}-\mathrm{m}^{2}\right]$} \\
\hline$I_{x x}=0,02358052$ & $\mathrm{I}_{\mathrm{xy}}=0$ & $\mathrm{I}_{\mathrm{xz}}=1,25711 \mathrm{e}-3$ \\
\hline $\mathrm{I}_{\mathrm{yx}}=0$ & $\mathrm{I}_{\mathrm{yy}}=0,2343418$ & $\mathrm{I}_{\mathrm{yz}}=0$ \\
\hline $\mathrm{Izx}=1,25711 \mathrm{e}-3$ & $\mathrm{I}_{\mathrm{zy}}=0$ & $\mathrm{I}_{\mathrm{zz}}=3,4191 \mathrm{e}-3$ \\
\hline \multicolumn{3}{|c|}{ Inercia Muñeca $\left[\mathrm{kg}-\mathrm{m}^{2}\right]$} \\
\hline $\mathrm{I}_{\mathrm{xx}}=1,62923 \mathrm{e}-3$ & $\mathrm{I}_{\mathrm{xy}}=0$ & $\mathrm{I}_{\mathrm{xz}}=0$ \\
\hline $\mathrm{I}_{\mathrm{yx}}=0$ & $\mathrm{I}_{\mathrm{yy}}=1,53621 \mathrm{e}-3$ & $\mathrm{I}_{\mathrm{yz}}=0$ \\
\hline$I_{z x}=0$ & $\mathrm{I}_{\mathrm{zy}}=0$ & $\mathrm{I}_{\mathrm{zz}}=8,9634 \mathrm{e}-4$ \\
\hline \multicolumn{3}{|c|}{ Inercia Mano (Grip) [kg-m²] } \\
\hline $\mathrm{I}_{\mathrm{xx}}=0,101940$ & $\mathrm{I}_{\mathrm{xy}}=-8,9 \mathrm{e}-7$ & $\mathrm{I}_{\mathrm{xz}}=0$ \\
\hline $\mathrm{I}_{\mathrm{yx}}=-8,9 \mathrm{e}-7$ & $\mathrm{I}_{\mathrm{yy}}=1,13936 \mathrm{e}-3$ & $\mathrm{I}_{\mathrm{yz}}=0$ \\
\hline$I_{z x}=0$ & $\mathrm{I}_{\mathrm{zy}}=0$ & $\mathrm{I}_{\mathrm{zz}}=3,3047 \mathrm{e}-4$ \\
\hline
\end{tabular}
ROTACIÓN GENERADOS CON SOLIDWORKS

Fuente. Elaboración propia.

\section{RESULTADOS}

El manipulador RV-M1 pertenece al grupo de robots industriales tipo vertical que se desplazan libremente en su espacio de trabajo realizando movimientos sin interactuar con el medio [15], ideal para tareas cinemáticas como pintado, corte y soldadura [1]. Para este tipo de robots el control de posición pura es adecuado [1], es decir, donde se requiere que cada articulación inicie su 


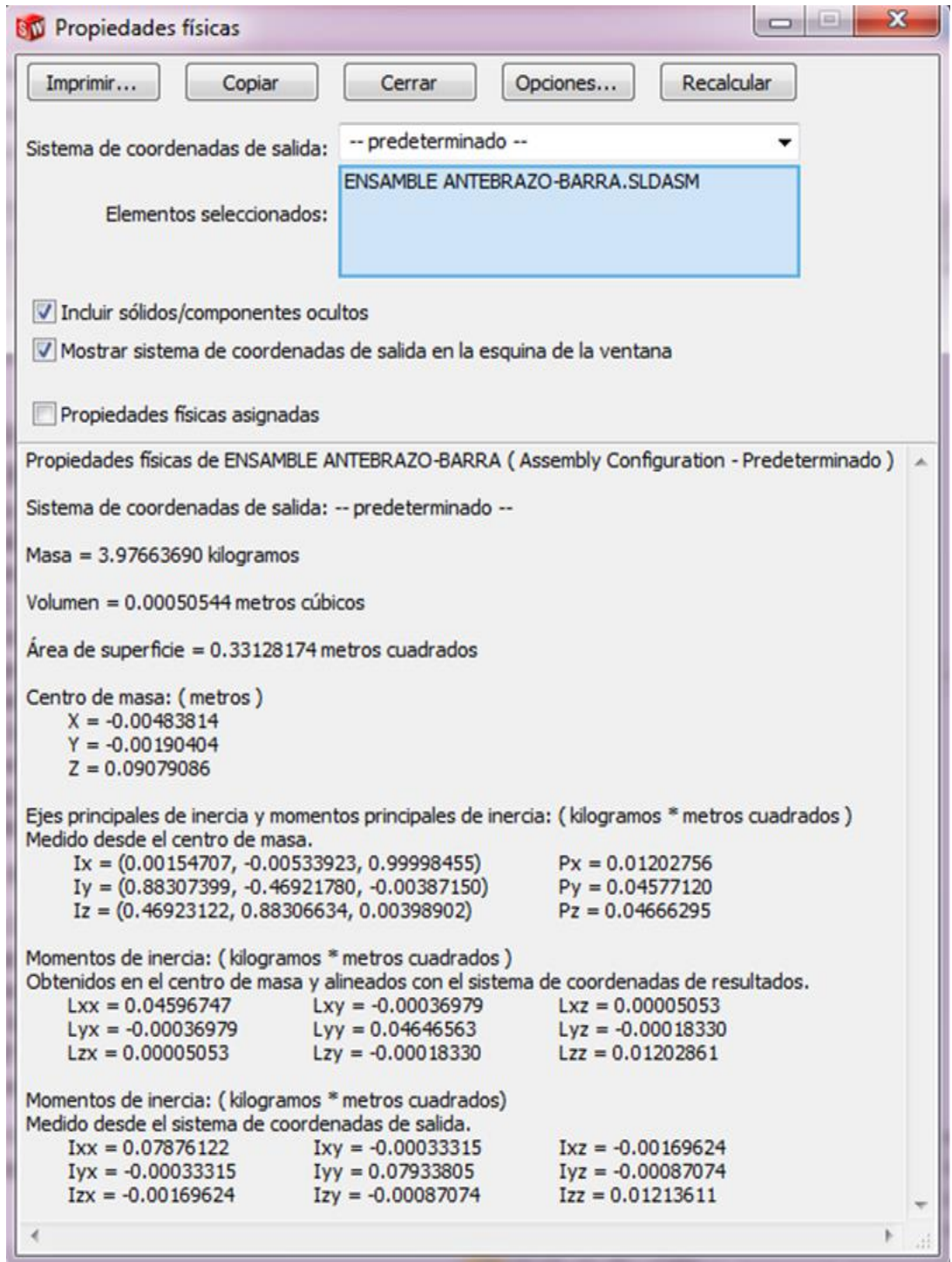

Figura 7. Valores de inercia arrojados por SolidWorks para el antebrazo, elemento 2.

Fuente. Elaboración propia

movimiento en una posición inicial de homming para alcanzar exactamente una posición final deseada. La ley de control escogida para simulación de compensación por gravedad es adecuada en este caso.

Consideraciones como que los actuadores del manipulador son ideales y no modifican el modelo dinámico han probado su efectividad en la implementación de la ley de control [19] al combinarlo con ajuste tipo tunning de las ganancias. Con esta consideración de actuadores ideales y asumiendo que los eslabones son rígidos y sin fricción en sus uniones, la dinámica del manipulador serial RV-M1 se puede modelar como la ecuación 37.
La figura 8 ilustra el esquema de la ley de control. El cálculo del par $\tau$ en la ecuación 43 requiere de una función vectorial no lineal en q y $\dot{\mathrm{q}}$, cuya representación establece la ley de control [1] mediante

$$
\tau=\mathrm{K}_{\mathrm{P}} \mathrm{e}(\mathrm{t})+\mathrm{K}_{\mathrm{D}} \dot{\mathrm{e}}(\mathrm{t})+\mathrm{G}
$$

en donde se han introducido las ganancias proporcional $\mathrm{K}_{\mathrm{P}}$, y derivativa $\mathrm{K}_{\mathrm{D}}$, ambas de tamaño $5 \times 5$ en este caso, las cuales tratan de llevar a cero los errores de posición e, y de velocidad è, definidos por las ecuaciones 45 y 46 , respectivamente. Las matrices de ganancias $\mathrm{K}_{\mathrm{P}}$ y $\mathrm{K}_{\mathrm{D}}$ son matrices simétricas definidas positivas. 


$$
\begin{aligned}
& \mathrm{e}(\mathrm{t})=\mathrm{q}_{\mathrm{R}}(\mathrm{t})-\mathrm{q}(\mathrm{t}) \\
& \dot{\mathrm{e}}(\mathrm{t})=\dot{\mathrm{q}}_{\mathrm{R}}(\mathrm{t})-\dot{\mathrm{q}}(\mathrm{t})
\end{aligned}
$$

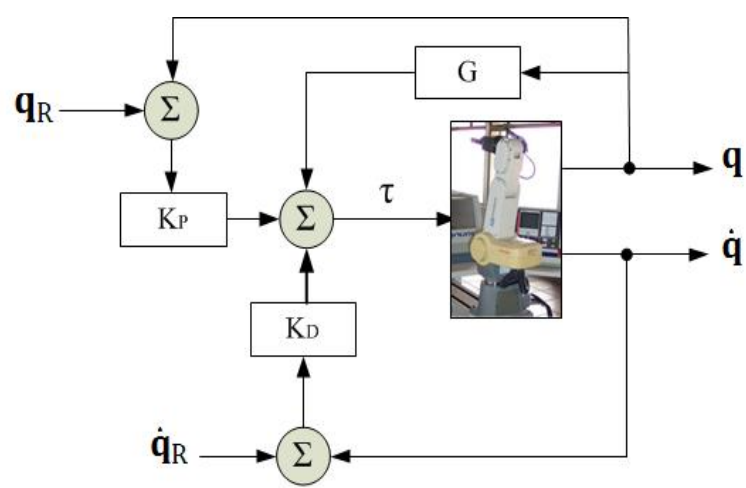

Figura 8. Estrategia de control por compensación por gravedad. Fuente. Elaboración propia.

Para ilustrar la metodología propuesta, se incluye una simulación de la estrategia de control de posición de compensación por gravedad, en la cual la herramienta del robot cambia de una posición inicial mostrada en la figura 9 (a) a una posición final mostrada en la figura 9 (b). Los ángulos de posición para cada junta se presentan en la figura 10, para un tiempo de simulación de 1 segundo.

Se diseñó un modelo en Simulink para el control por compensación por gravedad con el controlador mostrado en la figura 11. La planta se simula creando un subsistema con el bloque Subsystem de Simulink, especificando los elementos del manipulador, las entradas de torque y salida de posición y velocidad. Las propiedades de inercia asignadas a cada elemento fueron tomadas de la tabla II.

Con los valores de las ganancias proporcional y derivativa establecidos en $\mathrm{K}_{\mathrm{P}}=\operatorname{diag}([18,56,20,40$, 6]) 27 y $\mathrm{K}_{\mathrm{D}}=\operatorname{diag}([2,8,5,2,1]) 8$, respectivamente para un único controlador, se comparan las salidas para cada modelo dinámico: uno con los parámetros dinámicos hallados en este trabajo, y el otro con los usados por [5]. Tales parámetros dinámicos son las ubicaciones de los centros de masa, las masas de los eslabones utilizados y los elementos de las matrices de inercia. Las figuras $12 \mathrm{y}$ 13 presentan los errores en los espacios articular y de la tarea, respectivamente.

Los errores en el espacio articular son mostrados en la figura 12 para los ángulos de posición $\theta_{2}$ para el hombro (a), $\theta_{3}$ para el brazo (b), y $\theta_{4}$ para el antebrazo (c). Se observa que en los tres casos el error de posición angular es menor en la simulación correspondiente al modelo dinámico propuesto en este trabajo, denotado como "Proposed model". Continuando con el espacio de la tarea, la figura 13 presenta los errores de posición de la herramienta para las coordenadas x, y e z, en las figuras
15 (a), 15 (b) y 15 (c), respectivamente. Se aprecia que, excepto para el error en la coordenada x, el error de posición obtenido con el desarrollo presentado en este trabajo es menor al obtenido con los parámetros de [5].

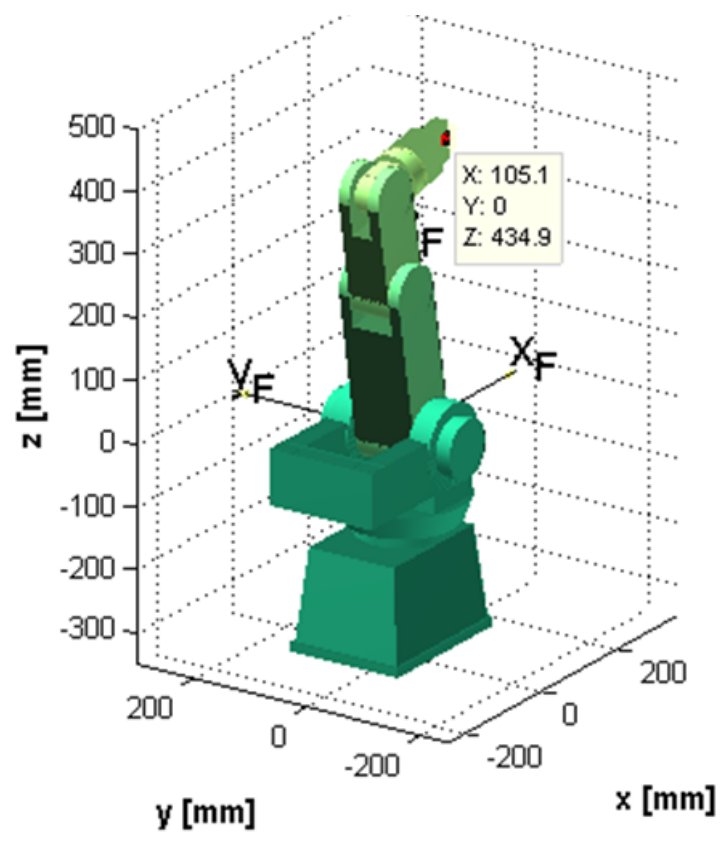

(a) Posición inicial

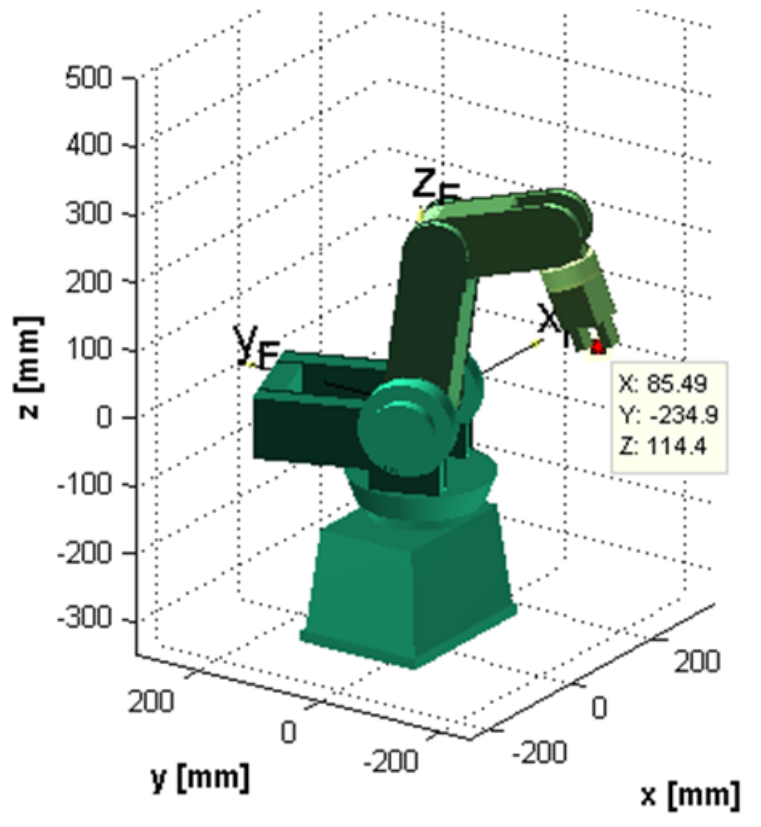

(b) Posición final

Figura 9. Posiciones (a) inicial y (b) final para la simulación. Fuente. Elaboración propia. 


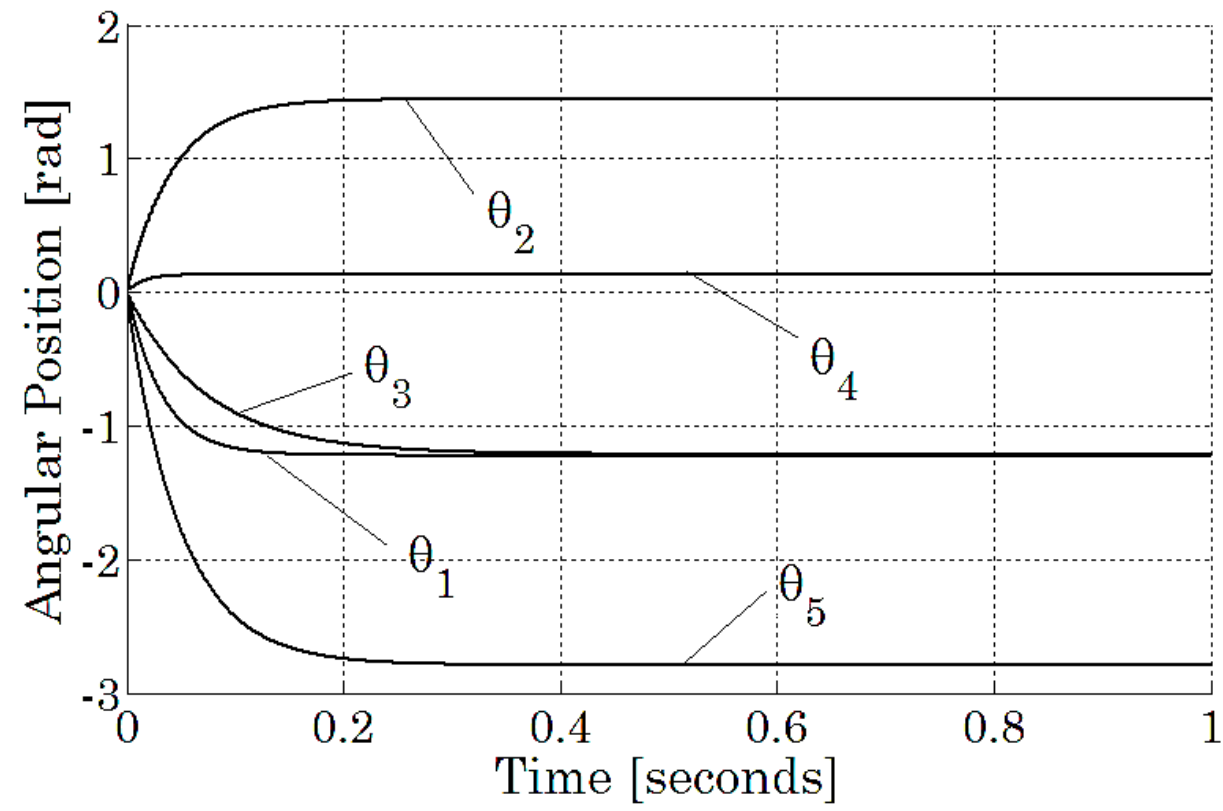

Figura 10. Ángulos de posición para la simulación. Fuente. Elaboración propia

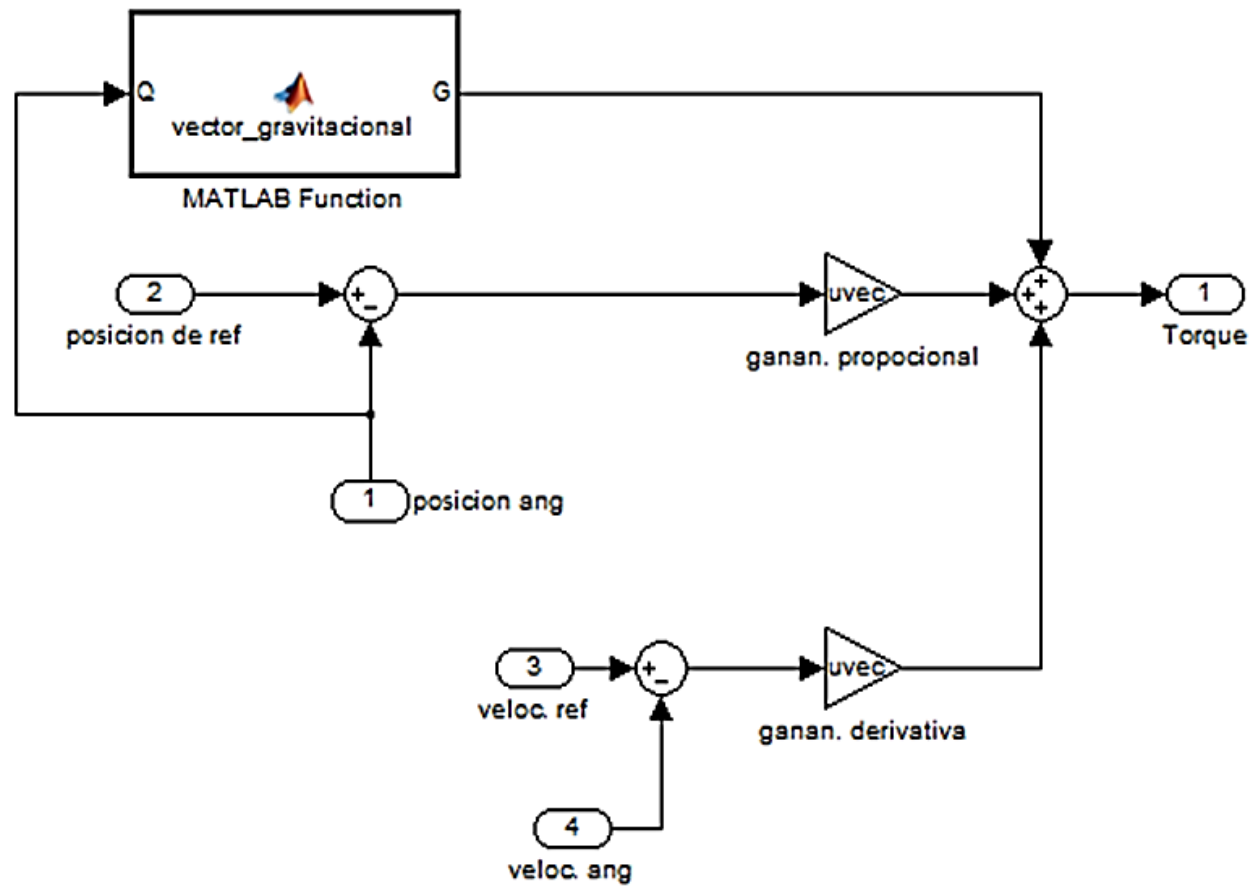

Figura 11. Bloque en Simulink del controlador de compensación por gravedad. Fuente. Elaboración propia. 


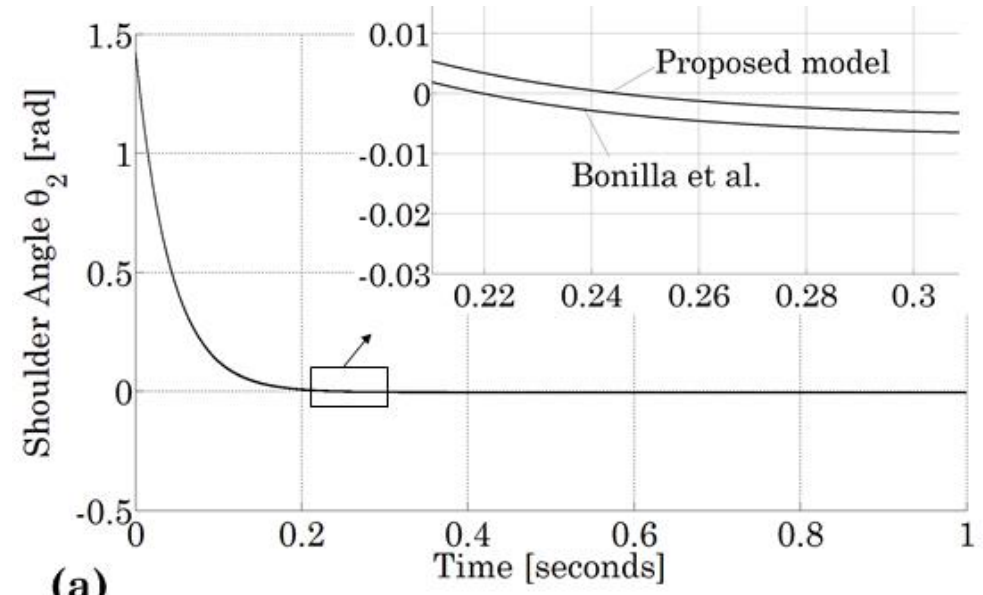

(a)

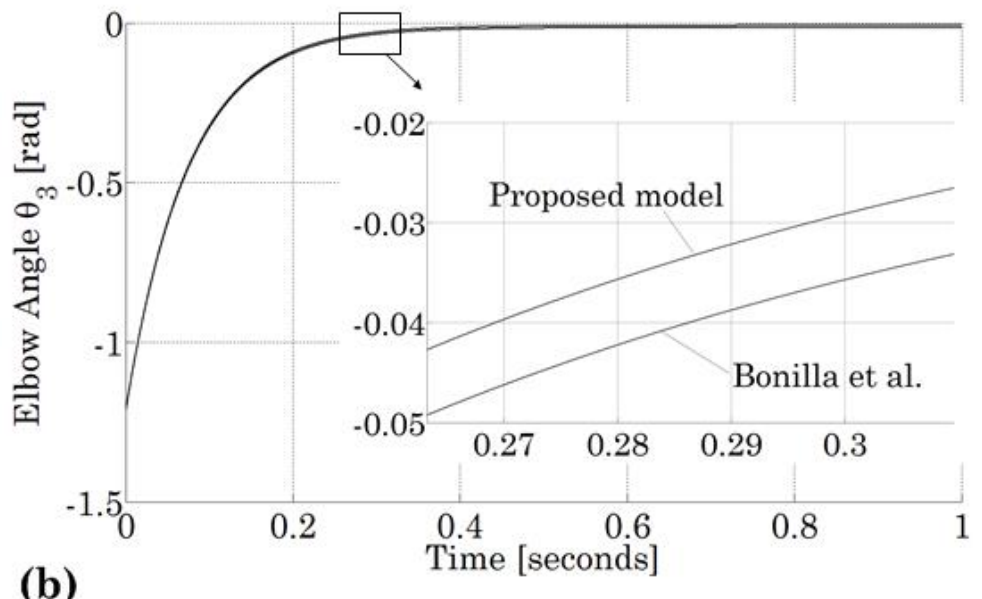

(b)

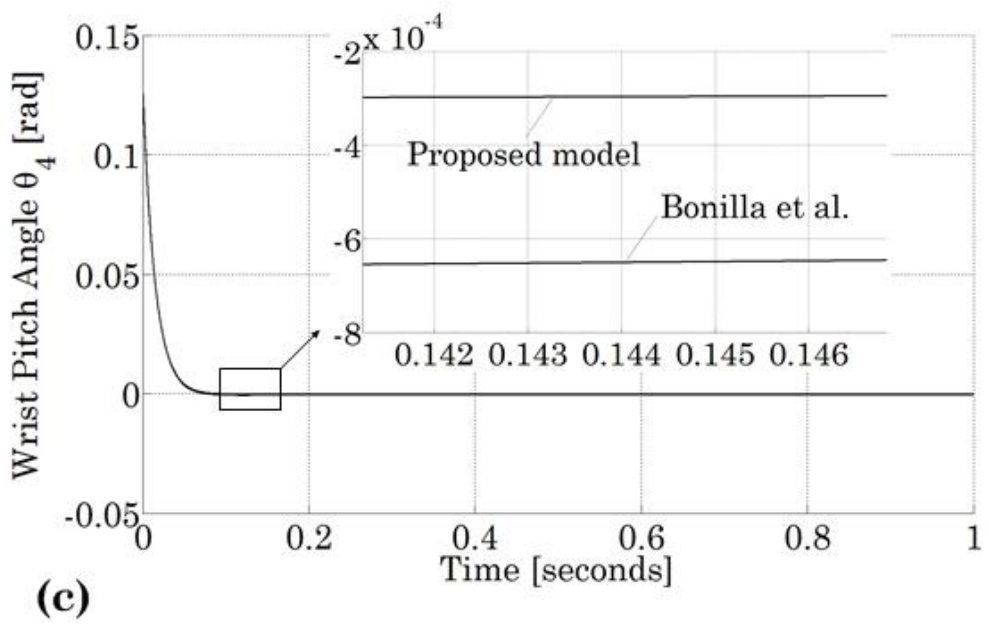

Figura 12. Comparación del error en el espacio articular para los elementos (a) segundo u hombro (b) tercero o codo (c) cuarto o muñeca. Fuente. Elaboración propia. 

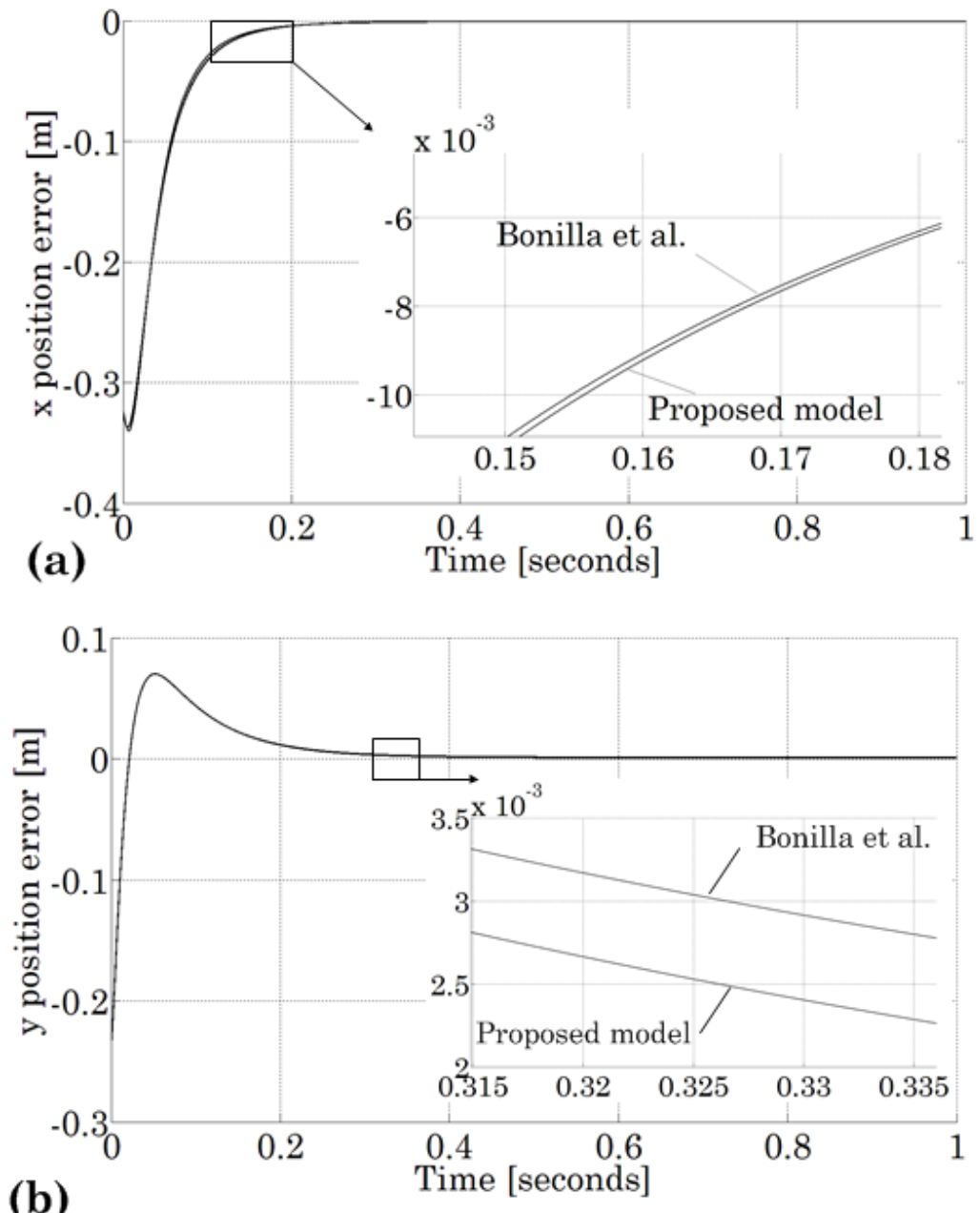

(b)

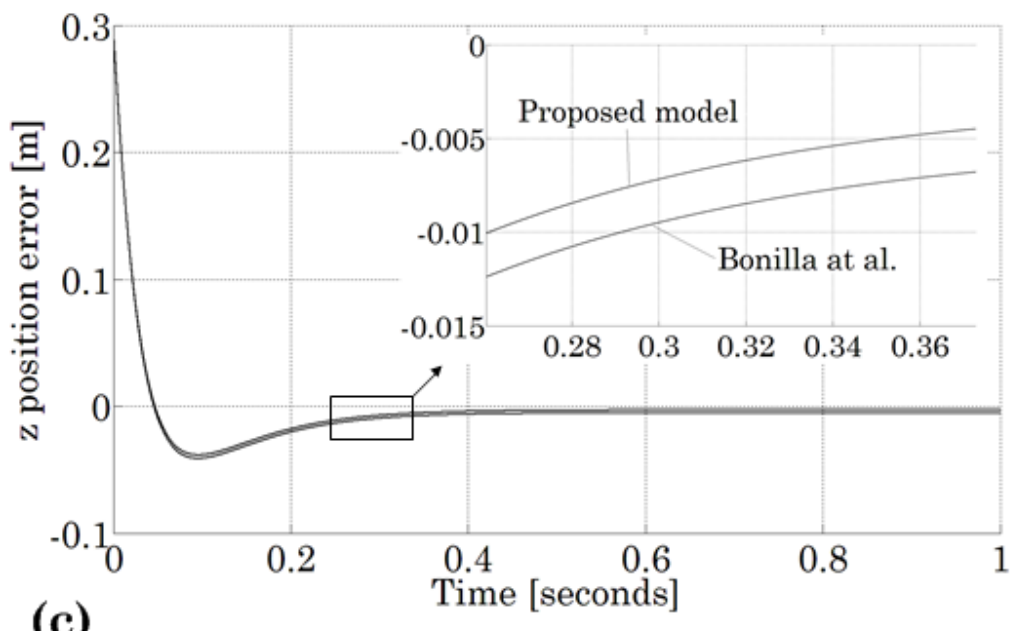

Figura 13. Comparación del error de posición en el espacio de la tarea para los ejes (a) x (b) y (c). Fuente. Elaboración propia. 
Dado que únicamente el error para la coordenada $\mathrm{x}$ en el espacio de la tarea es menor con los parámetros de [5], los resultados en general se consideran satisfactorios. La explicación para los errores más pequeños obtenidos con este modelo, bien se puede atribuir al nivel de elaboración del modelo CAD de cada eslabón móvil, incluyendo sus elementos internos

\section{CONCLUSIONES}

Se presentó la determinación de los parámetros dinámicos del robot Mitsubishi Movemaster RV-M1 usando SolidWorks con un modelo CAD que tuvo en cuenta los elementos internos constitutivos de cada elemento del robot. Un modelo matemático de la dinámica del robot fue desarrollado con base en el principio energético Lagrange-Euler y usado para estimar el torque requerido en la acción de control. La planta del robot fue simulada con el toolbox Simulink, también alimentada con los valores de ubicación de los centros de masa, masas, y matrices de inercias obtenidas. Se simuló la ley de control proporcional-derivativa de compensación por gravedad, al pasar el robot de una posición inicial a una final.

La simulación permitió la comparación del desempeño del robot con otros parámetros previamente reportados. En general, se puede colegir que tanto en el espacio articular como en el de la tarea los errores son menores al utilizar los parámetros encontrados en este trabajo, producto de un modelo CAD de alto nivel de elaboración y detalle. El mérito de este trabajo radica en que se utilizó un software no especializado en dinámica de maquinaria, se utilizó SolidWorks el cual es un software de Diseño de Maquinaria, muy común en las Facultades de Ingeniería.

Como trabajo futuro inmediato se propone la simulación de otras estrategias de control dinámico PID que permitan una comparación más amplia de resultados y llegar a generalizar la importancia de los parámetros encontrados.

\section{AGRADECIMIENTOS}

Los autores agradecen a la Vicerrectoría de Investigaciones, Extensión y Proyección Social de la Universidad del Atlántico por los recursos empleados en la elaboración de este proyecto.

\section{REFERENCIAS}

[1] R. Kelly y V. Santibañez, Control de Movimiento de Robots Manipuladores, $1^{\mathrm{a}}$ ed. Madrid, España: Prentice Hall, 2003.
[2] R. Kumar, P. Kalra y N. Prakash, "A virtual RV-M1 robot system", J. of Rob. and Comp.-Integ. Manuf., vol. 27, pp. 994-100, jun. 2011. DOI: http://dx. doi.org/10.1016/j.rcim.2011.05.003

[3] C. Hamilton, "Using MATLAB to advance the robotics laboratory", J. of Comp. Appl. in Eng. Edu., vol. 15, no. 3, pp. 205-213, Sep. 2007. DOI: 10.1002/cae.20143

[4] J. Craig, Introduction to Robotics: Mechanics and Control, $2^{\text {nd }}$ ed. New York, USA: Addison-WesleyLongman, 1989.

[5] M. Bonilla, V. Parra y F. Ruiz, "Co-Simulation of Cooperative Robots Based on ADAMS, MATLAB and a Haptic Interface", en Congreso Anual de la AMCA, (Puerto Vallarta, Jalisco, México), 2010. [En línea]. Disponible en: https://goo.gl/5cDXRQ

[6] MSC Software Corp., CA, EEUU. Adams. The Multibody Dynamics Simulation Solution. [En línea]. Disponible en: http://www.mscsoftware.com /product/adams

[7] M. Díaz et al, "Identificación de parámetros dinámicos de robot paralelos a partir de la medición del par y la posición en los actuadores", Rev. Téc. Ing. Univ. Zulia, vol. 32, no. 2, pp. 119-125, ago. 2009. [En línea]. Disponible en: http://www.scielo .org.ve/pdf/rtfiuz/v32n2/art04.pdf

[8] C. Wang, X. Yu y M. Tomizuka, "Fast Modeling and Identification of Robot Dynamics Using the Lasso", ASME 2013 Dyn. Syst. and Control Conference, (Palo Alto, CA, EEUU), 2013. DOI: 10.1115/DSCC2013-3767

[9] W. Verdonck, J. Swevers y J.C. Samin, "Experimental robot identification: Advantages of combining internal and external measurements and of using periodic excitation", J. Dyn. Sys., Meas., Control, vol. 123, no. 4, pp. 630-636, feb. 2001. DOI: $10.1115 / 1.1409936$

[10] J. Duffy y J. Rooney, "A foundation for a unified theory of analysis of spatial mechanisms", Journ. of Eng. for Ind., Trans. ASME, vol. 97, no. 4, series B, pp. 1159-1164, nov. 1975. DOI: $10.1115 / 1$ .3438718

[11] J. Duffy y C. Crane, "A displacement analysis of the general spatial seven link, 7R mechanism", Mech. and Mach. Theory. vol. 15, pp. 153-169, dec. 1980. DOI:10.1016/0094-114X(80)90001-4

[12] C. Crane, J. Duffy y T. Carnahan, "A kinematic analysis of the space station remote manipulator system", J. of Rob. Syst., vol. 8, no. 5, pp. 637-658, oct. 1991. DOI: $10.1002 /$ rob.4620080505 
[13] C. Crane, J. Duffy y T. Carnahan, "A kinematic analysis of the modified flight telerobotic servicer manipulator system", J. of Rob. Syst., vol. 9, no. 4, pp. 461-480, jun. 1992. DOI: 10.1002/rob .4620090403

[14] C. Crane y J. Duffy, Kinematic Analysis of Robot Manipulators, $1^{\text {st }}$ ed. NY, EEUU: Cambridge, 1998.

[15] Industrial Micro-Robot System User ManualModel RV-M1, Mitsubishi Electric Corporation, Naguya, Japan, 1992.

[16] R. Jazar, Theory of Applied Robotics: Kinematics, Dynamics and Controls, $1^{\text {st }}$ ed. NY, EEUU: Springer, 2007.

[17] J. Roldán et al, "Cinemática inversa matricial del manipulador 5R Mitsubishi Movemaster RV-M1", Rev. Épsilon, vol. 19, pp. 33-56. dic. 2012. [En línea] Disponible en: http://revistas.lasalle.edu.co /index.php/ep/article/view/2244

[18] L.W. Tsai, Robots Analysis: The Mechanics of Serial and Parallel Manipulators. New York, USA: Wiley Interscience, 1999.

[19] M. Takegaki y S. Arimoto, "A new feedback method for dynamic control of manipulators", Trans. of ASME, J. Dyn. Sys., Meas., Control, vol. 103 , pp. 119-125, jun. 1981. DOI: 10.1115 $/ 1.3139651$ 